\title{
Effect of Electrical Defloration in Liquid and Solid Crystals
}

\author{
Valentin. A. Tsvetkov \\ Problem Laboratory of Moscow State Academy of the Instruments Engineering and Computer Sciences, Moscow, Russia. \\ Email: tsvetkov_v_a@mail.ru \\ Received February $16^{\text {th }}, 2013$; revised March $16^{\text {th }}, 2013$; accepted March $24^{\text {th }}, 2013$ \\ Copyright (C) 2013 Valentin. A. Tsvetkov. This is an open access article distributed under the Creative Commons Attribution License, \\ which permits unrestricted use, distribution, and reproduction in any medium, provided the original work is properly cited.
}

\begin{abstract}
Earlier we observed a movement of the front of ions in the bulk of an ordered LC sample across which the driven voltage was applied [1]. This movement looked as waving zigzag curvatures in the pattern of interference strips. It was shown that behind the movable front of ions, the LC volume was getting charged. The author has found out that, in his pioneer experiments made in 1974, he observed the similar electrooptical phenomena on a single crystal of niobatestrontium-barium (NBS). Just after applying driving voltage (in a crossed electrical field crystal sample geometry) a system of curved interference strips is arising, which is evolving for the time period of about ten minutes at the driven voltage being kept constant. The evolution of the interference stripes pattern near positive, negative electrodes and in middle part of sample occurs in some different ways. At the end of evolution the system of strips disappears and the sample becomes practically homogeneous. The described process can be observed only once at the first applying of the driving voltage. This process is caused by redistribution of charges frozen at the crystallization and which are having an opportunity of the recombination at the first applying of the driving voltage. The obtained results are discussed with the possible applying the given experimental technique for solid and LCs parameters studying.
\end{abstract}

Keywords: LC; Carrier; Volumetric Charge; Ferroelectric Solid Crystal

\section{Introduction}

During some time we were engaged in studing movement of charged carriers in ordered bulk samples of nematic liquid crystals (LC). The LC sample was placed in a parallelepiped-shape cavity of a size of $3 \times 8 \times 0.3$ $\mathrm{mm}$. The internal walls of the cavity (except two lateral sides) were rubbed in a horizontal direction for providing planar orientation. The lateral sides were covered with thin film copper electrodes that were processed for providing homeotropic orientation.

At the coordinated aligning action of all of the sides a rather perfect monocrystal texture was created in the parallelepiped volume, with monocrystal optical axis being directed in the horizontal direction. In the absence of driving voltage the texture of LC sample, observed at crossed polarizes, looked like a system of horizontal interference's strips, stipulated by variations in thickness Figure 1. When the driving voltage (for example, $60 \mathrm{~V}$ d.c.) was applied to the LC sample, waving zigzag curvatures in the pattern of interference strips were induced near the positive electrode, the front of which moved to the negative one with the velocity of about 6 $\mu \mathrm{m} / \mathrm{sec}$.

We have shown that the $\mathrm{LC}$ volume behind the waving front has a surplus volumetric charge, whereas the LC volume before the waving front was electrically neutral. After the waving front passes through the whole sample, all LC volume is getting volumetric charged. Under the driving voltage applied the sample texture looked like waving zigzag curvatures in the pattern of interference

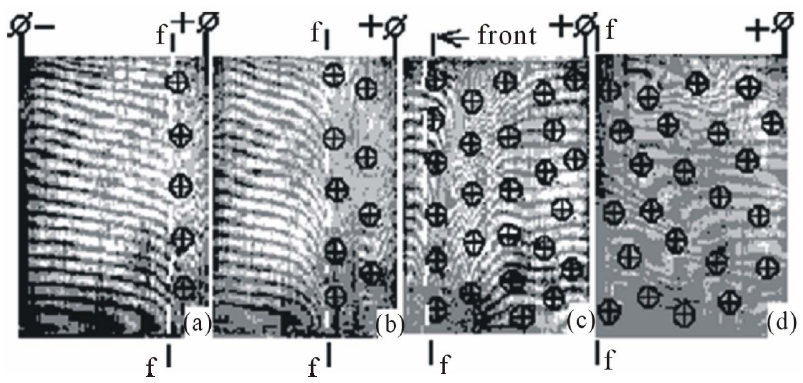

Figure 1. Successive frames of propagation of the wave of fringe distorsion. (a) 2 min after 60 voltage d.c. supply; (b) 3 min; (c) 8 min; (d) more than 30 min. 
strips. These phenomena are the result of local distortions of the external electrical field and of hydrodynamical stream flows both caused by moveable (driven) ions.

After the driving voltage is switched off, the curved interference strips are straightened and for the period of time of $\sim 1.5-2$ hours (optical relaxation time) they acquire an appearance of initial (undisturbed) interference pattern. The volumetric charge relaxes during neutral. After the waving front passes through the whole sample, all LC volume is getting volumetric charged. Under the driving voltage applied the sample texture looked like waving zigzag curvatures in the pattern of interference strips. These phenomena are the result of local distortions of the external electrical field and of hydrodynamical stream flows both caused by moveable (driven) ions.

After the driving voltage is switched off, the curved interference strips are straightened and for the period of time of $\sim 1.5-2$ hours (optical relaxation time) they acquire an appearance of initial (undisturbed) interference pattern. The volumetric charge relaxes during 10 - 12 hours (the charge relaxation time).

The described above processes can be repeated unlimited number of times provided that the volumetric charge is allowed completely to relax (i.e. the experiments may be repeated in the period of time not less than 12 hours).

The present work was carried out in the Microelectronics Department of Radio \& Electronics Institute (RAS), where the electrooptical properties of the solid ferroelectric monocrystal of noibat-barium-stroncium (NBS) were being studied by the neighboring team. So, we had decided to repeat the described above experiments with the sample of NBS-crystal.

\section{Investigations of Solid Crystals}

The sample represented a parallelepiped of a size of $1.5 \times$ $5 \times 15 \mathrm{~mm}$ with the optical axis being directed along the side of $1.5 \mathrm{~mm}$ Figure 2(a). All sides of the sample were carefully polished. On the lateral sides of the sample the metal electrodes (chromium) were deposited.

1) In the initial state being observed at crossed polarizes along the $5 \mathrm{~mm}$-size side, the sample had an appear ance of a homogeneous clear field. At applying $150 \mathrm{~V}$ d.c. to lateral sides (cross electric field mode of operation) interference strips were appeared in the optical pattern of the sample, indicating the presence of the electrooptical response for the inspected crystal. As the applied voltage changed the number of interference strips and their localization were essentially changed too. After the voltage switched-off the strips were disappeared and the sample pattern acquired an appearance of that at the initial state. Thus, the common cross electrooptical effect was ob- served.

2) We have found out that the electrooptical effect shows to some extent difference dependent upon whether the voltage is applied for the first time or it is done for the next ones. This difference consists of that at the first voltage applying there is a certain system of interference strips, which evolves for a long time. The various phases of this evolution are given in Figures 2(b)-(i). Just after the driving voltage applied, a system of narrow interference strips arises near the left (negative) electrode Figure 2(b) (area L).

In the other part of the sample (except area D) a system of relatively wide curved strips arises with the axis of total structure parallel to electrodes. In area D, apparently, there is a large defect of the crystal lattice not visible before applying the electric field. This defect distorting the local electric field is visualized just after the electric field is applied. After some time Figure 2(c) the strips in L-area are getting a little bit wider and their number is decreased. In the other sample part the number of strips are decreased, as well and they become smooth including the D-area

\section{Discussion}

The occurring processes can be interpreted as follows.

1) Just the external electrical field was applied to the dielectric sample, an internal electric field directed opposite to the external electric field was created, and electric current began to flow owing to the presence of carriers of different nature in the sample bulk. If the dielectric and resistivity properties of the sample material were strictly identical over the sample volume, the internal electric field and electro optical response would be identical in each point of the sample medium. Then the initially transparent sample would be blacked out, clarified or parallel straight interference strips would arise, depending on the magnitude of driving voltage and on the sample thickness. This picture would not be changed all the time, while the electric field was kept constant in magnitude. In our experiment interference strips are not straight and there are groups of strips in different parts of the sample that have a character look. The strips evolve for a long time, with the external electrical field being kept constant all this time. This phenomenon allows us to assert that the local electric field in different parts of the sample is nonuniform and its nonuniformity degree is changed in time. The local electric field is determined by dielectric constants and currents of carries through the sample, though in dielectrics the last are extremely small. It is hard to assume that the dielectric constants of the solid mono-crystal essentially differ over the sample volume, or even more so, they are changed while the external electric field is applied. Thus, the only reason of non- 

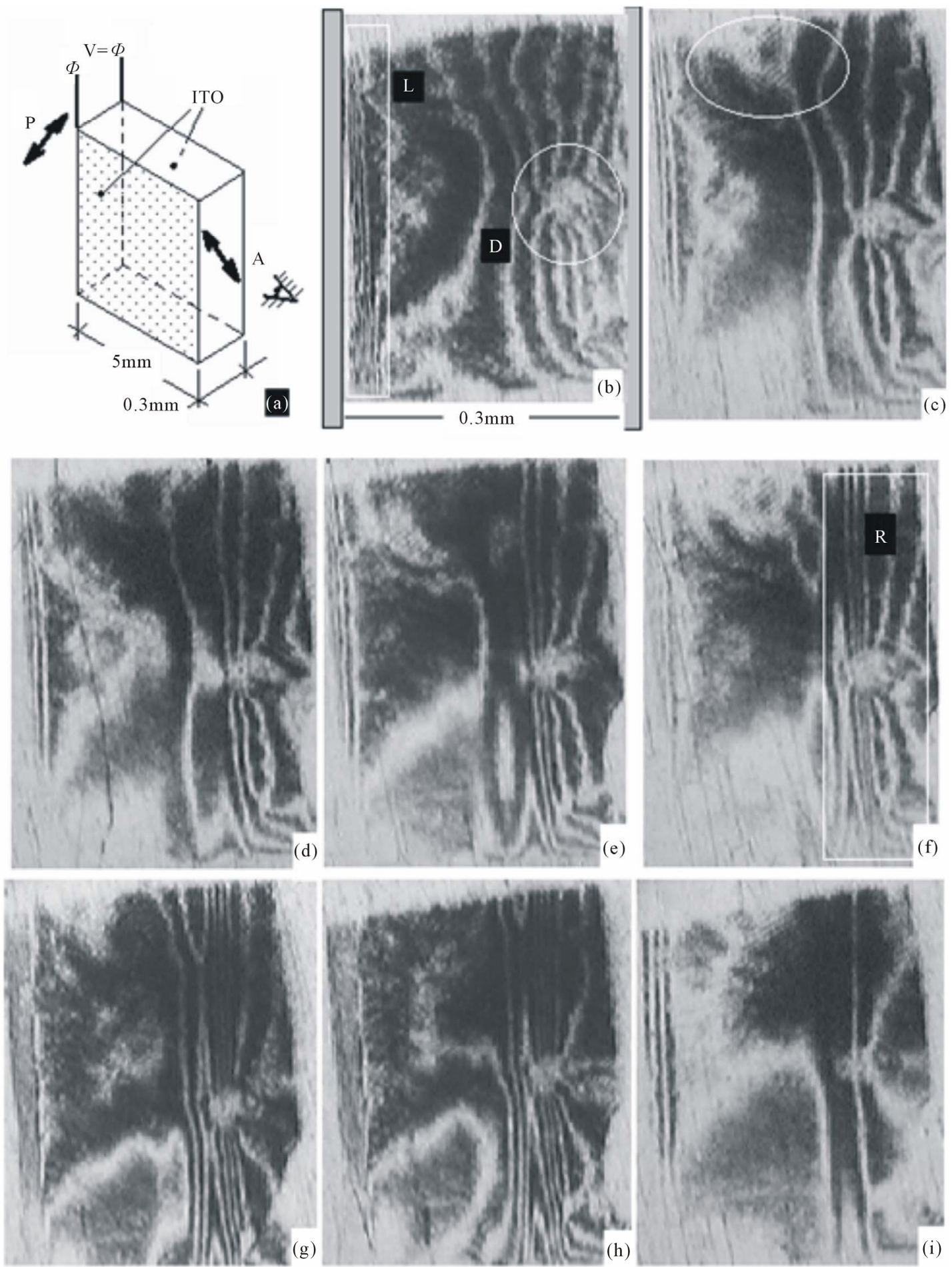

Figure 2. Sketch of sample and successive frames of evolution of interferencion strips. (a): sketch of sample; (b): 5 min after applying $50 \mathrm{~V}$ d.c.; $(\mathrm{c}(\mathrm{d}, \mathrm{e}, \mathrm{f}, \mathrm{g}, \mathrm{h}, \mathrm{i})): 10(20,35,45,55,>60)$ min after applying $50 \mathrm{~V}$ d.c.

uniformity of the local electric field and its value changing at the constant external electric field applied is unequal in value current caused by non-uniform distribution of carriers in different parts of the sample.

Let's consider the equivalent electrical circuit of the sample (one pipe of a current) as a circuit in series connected resistances $R_{L}, R_{m 1}, R_{m 2}, R_{R}$. The resistances $R_{L}$, $\mathrm{R}_{\mathrm{R}}$ characterize, accordingly, the left (negative) and the right (positive) adjacent electrode areas, and the resistance $\mathrm{R}_{\mathrm{m} 1}, \mathrm{R}_{\mathrm{m} 2}$, characterize resistance of the middle part 
of the sample. A current in the dielectrics, though also very small, is caused by carriers, which might be, in principal, free electrons, holes, drifting through the defects of the ionic crystal lattice, and in ferroelectrical crystals there are also charge carriers captured by domain boundaries.

During the process of monocrystal growth from a crystallizing crystal melt, an ordered crystal lattice is formed. The main problem of the monocrystal growth is the correct distribution of ions in the points of crystal lattice with possibly minimal number of failures. The problem of the local electrical neutrality for the formed lattice is not any way controlled and is solely a spontaneous process. It is rather probable, that to the moment of formation of following crystal layers there is a surplus or lack of electrons in some crystal lattice points of the previous layers and this charges distribution appears to be frozen in a grown crystal sample. Thus, in a crystal bulk chaotically located centers can exist (and does exist, as this is experimentally proved), that capture an excess (overbalance) of electrons or/and holes .The carriers can also be accumulated near the borders of domains of a ferroelectrical crystal, what the NBS sample studied actually is. And, in different parts of the sample there can simultaneously exist an excess of both free electrons and holes that are not recombine with each other because of significant distances between places of their localization.

If an external electric field is applied to such a ferroelectric crystal (represented electrically a conglomerate of domains and defects with chaotically distributed free charges), then electrons will begin to be injected from the negative electrode into the crystal bulk. One portion of the injected electrons will recombine with the hole excess in the L-area just adjacent to the negative electrode, the other one forms a bulk overbalanced negative charge in the same near-electrode L-area.

2) Near the positive electrode a depletion region of electrons is formed, as some overbalanced electrons are rejected into the positive electrode, and others can recombine with moveable free electrons, which can move from the middle crystal layers to the positive electrode area when the external electric field is applied.

As in the initial state the distribution of carriers over the crystal bulk is non-uniform, the resistance magnitude of $R_{m 1}, R_{m 2}, R_{R}$ for equidistant parts (from electrodes) of the sample are different. It results in different potentials for equidistant sample points and, as consequence, different optical phase shift along the equidistant sample lines. That's why the interference strips are bent.

3) In initial state, in the middle part of the sample there are charged carriers non-recombined by different reasons. After applying the electric field the free charged carriers begin to move: electrons are moving to the positive electrode, holes are moving to the negative electrode. During this counterpart carriers movement some of them may recombine that can not be done before the electric field applying. As a result, the amount of the electrical nonhomogeneities in the middle part of the sample is decreased and crystal bulk is getting more homogeneous electrically. In any case the resistance distribution pattern of the crystal volume is changed. This leads to the redistribution of the electric potentials in the sample volume in accordance with the new resistance values of $R_{L}, R_{m 1}$, $R_{m 2}, R_{R}$. As the result of the potential redistribution in the sample bulk, new interference strip patterns arise, which are presented in the all-subsequent photos Figures 2(c)(i). The described above processes last during the long period of time because of very small magnitudes of mobility of charged carriers that take place in a good insulator the NBS-crystal really is.

4) In boundary areas, especially in the L-layer adjacent to the negative electrode, under the electric field applied, there is an area having the pattern of plenty of narrow interference strips weakly changing with time. It is clear that free electrons exceedingly saturate this area and such a state is very stable. That's why, the interference strip pattern near the electrodes is small unchangeable in time.

In the R-area, near the positive electrode, for the all period of the electric field applied there is also a system of interference strips, which wider than those near the negative electrode, and the whole system itself is greatly wider than that in the L-area, near the negative electrode.

After the long period of time most of the carriers in the middle part of the sample recombine and the electric field is getting practically homogeneous. The interference pattern of Figure 2(i) corresponds to this state.

5) After the electric field is switched off, the interfereence pattern in the middle of the sample is disappeared in a fraction of second, for the negative electrode area it takes several minutes, and for the positive electrode area it lasts more than several days. The last circumstance is due to the difficulties of volume charge relaxation that takes place in a good dielectric with very low carrier mobility. Even for the poor dielectric, what LC is, the volume charge relaxation time is about of $10-12$ hours for sample geometry close to described one [1].

6) The most interesting is that the second and all subsequent application of the electric field of the same voltage and the same polarity to the completely relaxed sample, a system of interference strips arise that is similar to that of Figure 2(i), but not to Figure 2(b). Switching on the electric field of the same voltage but the opposite polarity results in formation of interference pattern distinguished from that of Figure 2(i) by exchanging the places of the L- and R-areas, with the interference pattern in the middle of the sample being kept practically un- 
changeable. It confirms the fact that at the first application of the external electric field there occurred an ireversible change of the electrical condition in the volume of the sample.

By analogy to beautiful process, known in an alive nature it is possible to name the observed electrooptical phenomena as "electrical defloration".

7) The mobility of carriers in LCs is rather large for the sample to relax to the initial state completely even if the relaxation process lasted for long time. In solid crystals the mobility of carriers is small. Therefore, up to the first driving electric field application to a crystal sample, chaotically dispersed over the crystal bulk carriers had no chance to recombine, except the couples for which carriers were very close to each other. But after switching-on and durable action of an external electric field, additional numbers of carrier couples have chances to recombine, that's why the sample volume is getting more homogeneous electrically. This energetic state is a stable one and there is no reason to return to the original state.

8) Owing to grate difference in carrier mobilities for LC's and solid crystals, electrical defloration process in LC's can be repeated an unlimited number of times. On the contrary, for solid crystals it is fundamentally a nonrecurring phenomenon that can occur only one time.

9) A usage of the carrier transport observation technique in LC's for solid electrooptical crystals allowed us to observe and explain the new electrooptical phenomenon for the last (named by us as "electrical defloration"). Besides, as it's seen in Figure 2(b) (area D), it is possible to observe process of homogenization of charges in the field of defects. In Figure 2(i) one can see, that at the end of defloration process there were left only small traces from originally existing large area D. It opens an opportunity for the further development of described technique for visualization and nondestructive inspection of defects in solid single crystals.

10) Another interesting phenomenon observed with the described technique is a formation of system of fine parallel interference strips which have a width size of about $40 \mu \mathrm{m}$. They are directed at the angle $\sim 45^{\circ}$ to the verticals in area $M$ during time from 5 mines up to 240 of mines Figures 2(b)-(i). Unfortunately, these strips are well seen only in the original photos or at the large optical increase.

It is quite possible, that here we observe a process of formation of monodomain ferroelectrical crystal (by the way, as far as we know, nobody observed domains in NBS as early as we had done).

At the beginning of the process one can not observe any stripes. It explains our first failure we had in our very early attempts to visualize expected domain structure for NBS-crystal by depositing onto the polished crystal surface or onto the crystal chip a thin LC-layer. It is the same technique, which allowed visualizing domains, for example, in triglycine sulfate-crystal (TGS) [2]. That time we had not seen any attributes of domain structure in NBS. And a conclusion was made that the domains are too small and outside the technique limit. Under the electric field applied, the domains, fine, invisible by any other techniques, with different directions of polarization, are polarized and form larger domains (recrystalization and monodomainization processes). Then, after some period of time of d.c. electric field action, they became visible Figures 2(c)-(e).

At the further reorganization process, the monodomain structure is practically formed.

We have paid attention to these fine strips in photos only at writing this paper and now we have no chance to repeat the experiment. Therefore, the interpretation of these strips as domain existing is one of the possible hypotheses not forbidden by nature, but also not confirmed precisely the domain existing. If those are domains, their sizes in the initial condition would be less than 40 $\mu \mathrm{m}$ and then, it is logical, that we could not to visualize them by putting onto the chip surface a LC layer of smaller thickness $(\sim 20-30 \mu \mathrm{m})$ than domains were of.

\section{Conclusions}

1) The experimental technique of observing electric field distortions induced by the moving charged carriers in a LC-medium under the constant electric field was used for studying the charged carrier movement in a solid ferroelectric crystal of niobate-strontium-barium. There are given the comparison of processes of transportation of charges carriers in LCs and solid crystal in close geometries of experiment. The analogies and distinctions of these processes are shown.

2) A noncontradicting-to-physical-laws explanation of electroptical phenomena arising near the anode, the cathode and in the middle part of the NBS-crystal sample is given on the basis of experimental studying the evolution of the interference pattern induced by the external electric field applied to the sample. In particular, single process of homogenization of electric nonhomogeneities formed in process of ferroelectric crystal growth was shown to occur in the middle part of the crystal sample.

It is obvious that at a lot of experiences with identical on thickness and for each experience by new (fresh) samples it is possible to receive the quantitative information on mobility of carriers in a solid crystal.

3) With the described technique there were observed processes of homogenization of electric near-defect nonhomogeneities. In future the technique can be advanced for the quality control of electrooptical crystals.

4) In the first the size of domains in NBS ferroelectric crystal is determined with the large fraction of prob- 
abiity. In the initial state they are of a size less than 40 $\mu \mathrm{m}$.

\section{Acknowledgements}

The author wish to thank Drs Zakharov L. and Ermakova (Phillipova) O. (Laboratory of Microelectronics of RAS) for the delivered NBS-sample and fruitful discussion, in spite of the fact that not on all questions we had identical opinion.

\section{REFERENCES}

[1] V. A Tsvetkov and O. V. Tsvetkov, "Ions Influence on Electrooptical Characteristics of NLC". Molecular Crystals and Liquid Crystals, Vol. 368, No. 1, 2001, pp. 625632. doi:10.1080/10587250108029995

[2] N. A. Tikhomirova, S. A. Pikin, et al., "Vizualization of domains in ferroelectricTGS crystals," Ferroelectrics, 29, 1980, pp. $1145-1156$. 